Classification

Physics Abstracts

$32.60 \mathrm{~F}-32.80$

\title{
Low field linear Stark effect on quasi-hydrogenic caesium series
}

\author{
C. Chardonnet, F. Penent, D. Delande, F. Biraben and J. C. Gay \\ Laboratoire de Spectroscopie Hertzienne de l'E.N.S. (*), \\ Tour 12, E 01, 4, place Jussieu, 75230 Paris Cedex 05, France
}

(Reçu le 25 mars 1983, accepté le 6 mai 1983)

\begin{abstract}
Résumé. - Nous présentons la mise en évidence expérimentale de l'effet Stark linéaire dans des conditions de champ électrique faible (en valeur absolue), sur des séries presque hydrogénoïdes du caesium atomique. C'est le comportement qui serait observé sur l'hydrogène pour l'ensemble du spectre dans les mêmes conditions. On le met en évidence ici, dans des champs électriques de 1 à $15 \mathrm{~V} / \mathrm{cm}$, sur des atomes presque hydrogénoïdes excités en phase vapeur dans des états de Rydberg de valeurs de $n \simeq 60$, à l'aide d'un laser continu. Les effets du cœur de l'atome sont presque négligeables comparés au terme d'effet Stark linéaire dans la série de perturbation.

Abstract. - We present the experimental evidence of the linear Stark behaviour in an atomic spectrum at low (absolute) electric field strengths on high lying quasi-hydrogenic series of atomic caesium. This is the behaviour which would be observed on hydrogen atoms for any level. It is exhibited here in the $1-15 \mathrm{~V} / \mathrm{cm}$ range on quasi-hydrogenic atoms, excited, in vapour phase, by a c.w. dye laser to $n \simeq 60$ Rydberg states. Under these conditions, the core effects are almost negligible compared with the first linear-in-field term in the Stark expansion of the energy.
\end{abstract}

The study of the Stark effect on high Rydberg series has been a matter of considerable interest for the last five years [1-6]. This results in a completely renewed understanding of phenomena as basic as ionization.

The non-relativistic hydrogen atom problem in an electric field is separable in parabolic coordinates $(\xi, \eta) . L_{Z}$ (projection of the angular momentum on the field axis) and a generalized version $A_{Z}$ of the Lenz vector are still constants of motion. The existence of $A_{Z}$, responsible for the dynamical symmetry in the problem, leads to a general crossing behaviour in the Stark map for the hydrogen atom. The spectrum displays three characteristic regimes. For field values smaller than $E_{\mathrm{c}}=-1 /\left(16 n^{4}\right)$ (the so-called classical ionization field), it is a quasi-bound state spectrum since the external field is small compared to the Coulomb binding field. We are here essentially concerned with this first regime.

Indeed, practical experimental situations are limited to non-hydrogenic atoms for which close range corrections to the Coulomb potential are responsible for a breaking of the dynamical symmetry. Hence, the problem is no longer separable. Nevertheless, at large distances, one will

(*) Laboratoire associé au C.N.R.S. LA 18. 
asymptotically recover the hydrogenic behaviour. This is the basic remark for extending the treatment of the Stark effect to non-hydrogenic situations [7, 8]. For sufficiently large field values, the Stark spectrum will look like that for hydrogen as far as the general behaviour is concerned. But a general non-crossing rule is obeyed and the intensity spectrum will be completely different [7-9].

Presently, we are interested in the low-field situation. For the hydrogen atom, the eigenstates are labelled with zero-field parabolic quantum numbers $\left(n, n_{1}, n_{2}^{-}, M\right)$ with $n=n_{1}+n_{2}+|M|+1$. The field dependence of the energy is then (atomic units) :

$$
\varepsilon=-\frac{1}{2 n^{2}}+\frac{3}{2} n\left(n_{1}-n_{2}\right) E-\frac{n^{4}}{16}\left(17 n^{2}-3\left(n_{1}-n_{2}\right)^{2}-9 M^{2}+19\right) E^{2}+\cdots
$$

and on a wide range of electric fields, $\left(0<E \lesssim E_{\mathrm{c}}\right)$, linearly depends on the electric field strength (hence the spectrum is approximately $M$ degenerate). Such a feature is specific of the hydrogen atom and associated with the degeneracy of the zero-field Coulomb problem.

Our purpose is to show that, for non-hydrogenic atoms, such a regime can take place at the lowest field values provided the effects of non-Coulombic corrections are energetically negligible compared with the linear Stark term in (1). They are measured with a quantum defect $\delta$. The condition is then :

$$
\frac{1}{n^{3}} \delta \ll \frac{3}{2} n\left(n_{1}-n_{2}\right) E .
$$

$L_{Z}=M$ is conserved in the Stark mixing and $\delta$ only depends on $L$. Then the larger the $M$ and $n$ values, the smaller the electric field strenght for which (2) holds. The observation of the linear Stark effect is possible on many electron atoms, at low fields, provided one chooses almost hydrogenic situations and sufficiently high $n$ values. This opens the way to various kinds of studies [10].

\section{Low-field linear Stark effect on atomic caesium.}

We here report the experimental study of the linear Stark effect on quasi-hydrogenic high-lying Rydberg states of atomic caesium. The electric field values are in the range $1-15 \mathrm{~V} / \mathrm{cm}$ and about 500 times smaller than in previous experimental investigations [11], but the range of $n$ values is between $n=30$ and $n=120$. For this latter $n$ value, the classical ionization field is only $1.6 \mathrm{~V} / \mathrm{cm}$. This is presently the only experiment performed under vapour phase conditions having allowed the exhibition of the linear Stark dependence in the atomic spectrum. We limit this report to the analysis of the position of energy levels.

\section{Experimental set-up.}

The experimental apparatus is basically the same as in the studies of diamagnetism and quasiLandau resonances previously reported $[12,13]$. The optical excitation uses a $800 \mathrm{mw}$ power, single mode, c.w. dye laser ( $\mathrm{R} 6 \mathrm{G}-\mathrm{Ar}^{+}$pumped) with $10 \mathrm{MHz}$ linewidth. The laser frequency is locked on an external cavity allowing $150 \mathrm{GHz}$ single mode pressure scans. Wavenumber calibration to $\pm 0.01 \mathrm{~cm}^{-1}$ is obtained through comparison to the molecular iodine spectrum. The laser beam is focussed on the interaction region of a pyrex electrostatically shielded thermoionic detector containing pure caesium vapour at a pressure of $10^{-2}$ torr. The detector is of a conventional type [12] and designed in order to apply a static uniform electric field in the interaction region. The field plates are spaced about $0.5 \mathrm{~cm}$ apart, one of which being a mesh with $50 \%$ transparency. The tungsten wire is heated at $800 \mathrm{~K}$ and is located in the equipotential volume. No additional electric field is applied between the wire and the surrounding electrode. The stray electric field in the interaction region is smaller than $20 \mathrm{mV} / \mathrm{cm}$. 
The excitation scheme uses the hybrid-resonance process $[12,14]$ involving the non-resonant absorption of two photons in $\mathrm{Cs}_{2}$ dimers. The absorption of a first photon populates the $5^{2} \mathrm{D}_{3 / 2,5 / 2}$ states of atomic caesium. The absorption of a second photon with the same frequency allows an extremely efficient excitation of the $n \mathrm{P}$ and $n \mathrm{~F}$ Rydberg series of atomic caesium. The excitation of the $F$ series can be achieved between $n=20$ and $n=160$ [12]. The latter value is a natural upper limit for a non Doppler-free process as the spacing $2 R / n^{3}$ between successive members is of the order of the $1 \mathrm{GHz}$ linewidth. Such an excitation scheme nicely fits our purpose of a study at low fields in as wide as possible a range of $n$ values.

Practical limitations to the studies come first from the excitation of discharges in vapour. This occurs usually for field strengths of about $30 \mathrm{~V} / \mathrm{cm}$. It is not a drastic limitation since discrete lines are hardly seen for fields greater than $15 \mathrm{~V} / \mathrm{cm}$. As the classical ionization field is smaller than $30 \mathrm{~V} / \mathrm{cm}$ for $n>55$, it is likely that the important production of electrons will anyway cause a smearing out of the lines.

Another limitation, but at low fields, is the Doppler width. The various components of the Stark manifold will be resolved when the spacing $3 n E$ between two successive components is larger than the Doppler width. The relevant minimum $E$ value is then $n$ dependent.

The last limitation concerns the selection of the $M$ value in the optical excitation. The excitation of 5D atoms through molecular dissociation processes does not allow any selection of the intermediate Zeeman substates to occur. All are populated but inequally as shown in previous experimental studies [12] and confirmed in the present one. Practically, the transitions (5D, $M= \pm 2 \rightarrow n \mathrm{~F}, M= \pm 3$ ) are fairly dominant in $\sigma$ polarization to the field $\mathbf{E}$ while the (5D, $M= \pm 1 \rightarrow n F, M= \pm 1 ; n \mathbf{P} M= \pm 1$ ) are the most important ones in $\pi$ polarization. Note that, in contrast to the situation in diamagnetism [12], it is not possible to distinguish between the various $M$ values from the experimental Stark map at low fields.

Indeed, from (1), the spectrum is $M$ degenerate as far as the linear Stark regime is concerned. The $M$ dependence appears in the second order term and is a small one in the present range of electric field values. For $n=60, E=10 \mathrm{~V} / \mathrm{cm}^{\prime}$ and $n_{1} \simeq n_{2}$, it is $1.8 \times 10^{-4} \cdot M^{2} \mathrm{GHz}$. A more relevant analysis, for the $M= \pm 3$ states of caesium, including quantum defects, confirms this conclusion.

An important advantage in the previous situation turns out to be its quasi-hydrogenic character. Indeed, the Stark mixing of the $M= \pm 3$ states involves the zero-field states with $l$ values such that $l \geqslant 3$. Their quantum defects, measuring the departure from the pure hydrogenic behaviour, will consequently be smaller than the defect, $\delta(F)=0.033$, of the F state, and fairly small. As a result, at low field values and for $\sigma$ polarization of the laser beam, the experimental situation will be nearly hydrogenic. For a convenient choice of the $n$ value in equation (2), the Stark diagram will exhibit the famous linear-in-field dependence from the lowest electric values.

No spurious fine structure decoupling effects due to the external field are likely to exist. The fine structure is $99 \mathrm{~cm}^{-1}$ in the 5D states while only $19.3 \mathrm{MHz}$ for the $n=36 \mathrm{~F}$ states. Then, it is not decoupled in the lower state of the optical transition while completely decoupled at the lowest field values in the upper state.

Figure 1 displays the structure of the hydrogenic manifold for $n=36,|M|=3$ and $\mathbf{E}=11.3 \mathrm{~V} / \mathrm{cm}$. The manifold is split into 33 components with a spacing 3 n.E (in atomic units) as expected, and its average extension is about $3 n^{2} E$. The various lines are associated with the $\left(n, n_{1}, n_{2},|M|=3\right)$ states in electric field and are labelled in the manifold with the $\left(n_{1}, n_{2}\right)$ zerofield parabolic quantum numbers. This is the regime where $L^{2}$ breaks down as a good quantum number while $\left(n_{1}, n_{2}\right)$ are still approximately defined. As seen on the plot of figure 2 , the behaviour of the energy levels as a function of the field strength is linear and is fairly well described within the linear approximation in (1). At low fields, it is yet clear that the role of the quantum defects of the $F$ and $G$ states is not negligible and that condition (2) must be fulfilled before the energy spectrum behaves as in hydrogen. 


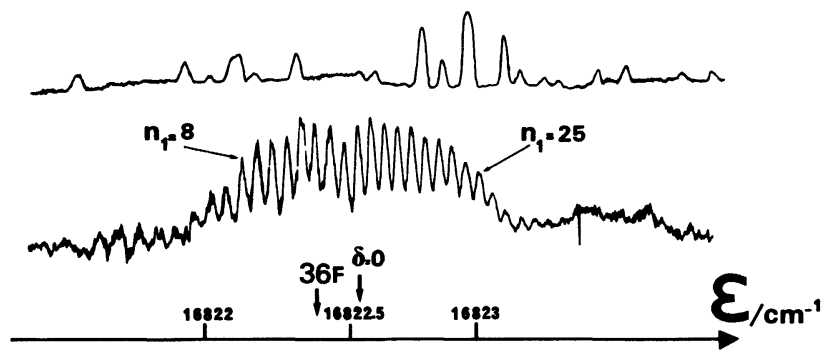

Fig. 1. - Quasi-hydrogenic structure of the $(n=36,|M|=3)$ manifold of atomic caesium in an electric field of $11.3 \mathrm{~V} / \mathrm{cm}$. The upper trace is the molecular iodine spectrum. The figure displays the 33 components of the manifold from $n_{1}=0$ to $n_{1}=32$. They are approximately equally spaced from a quantity $3 n E$ (atomic units) as expected from the hydrogenic approximation. (The energy scale is not linear).

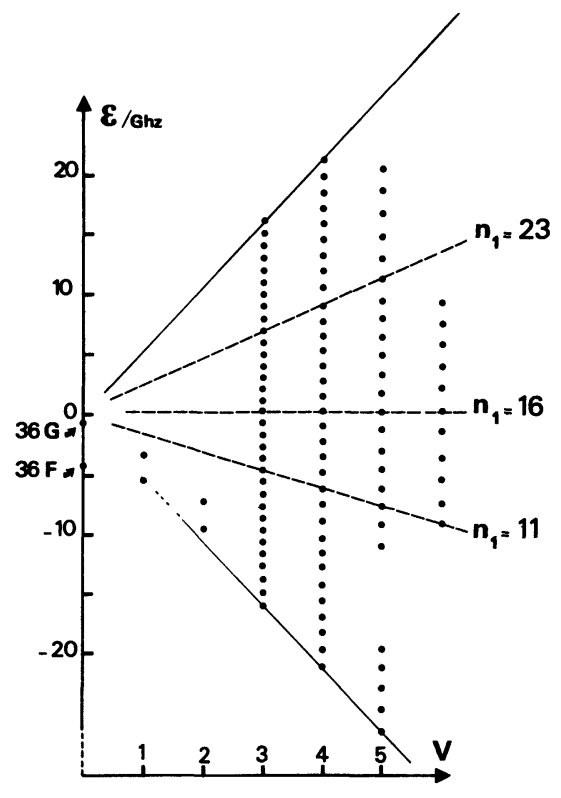

Fig. 2. - Stark map of the $(n=36,|M|=3)$ states of atomic caesium. At low fields, the role of the quantum defect of the $F$ and $G$ states is not completely negligible and the various lines cannot be resolved due to the Doppler resolution in the experiment. At higher fields, the behaviour is linear in $E$ field as expected and the various lines are equally spaced. The $\left(n_{1}=16, n_{2}=16,|M|=3\right)$ component does not exhibit any linear Stark effect as expected from (1). The field value is $E=2.3(\mathrm{~V} / \mathrm{cm})$.

For higher $n$ values and within the present range of electric field strengths, it is possible to observe the Stark map in condition where several adjacent manifolds are interacting, that is when $\frac{1}{n^{3}} \sim \frac{3}{2} n\left(n_{1}-n_{2}\right) E$. Such a plot is shown in figure 3 around $n=60$, in $\sigma$ polarization. There are two kinds of independent contributions in such a plot associated with $|M|=3$ states and $|M|=1$ states, respectively. Both classes of states are excited in the optical process but with different efficiencies. The Stark diagram associated with $|M|=3$ states only involves states with highly hydrogenic behaviour. The role of quantum defects is almost negligible as far as the energy 


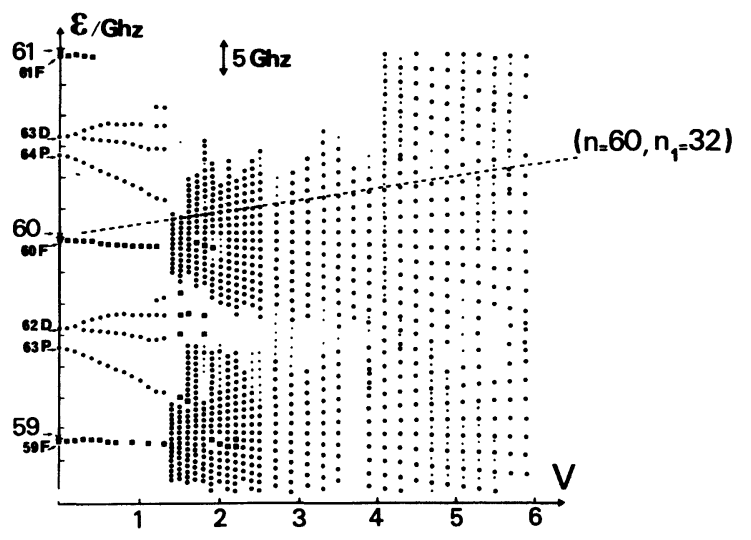

Fig. 3. - Stark map of atomic caesium around $n=60$. There are two independent contributions associated with $|M|=3$ quasi-hydrogenic states and $|M|=1$ non-hydrogenic states. $|M|=3$ states are clearly distributed around the $n=59,60,61$ zero field positions of the quasi-hydrogenic manifolds. The energies of all the components linearly behave with the electric field which is quite clearly seen in the figure. In the strong mixing regime (when $2 / n^{3} \sim 3 n\left(n_{1}-n_{2}\right) E$ ) the various components can still be tracked without any ambiguity meaning that the anticrossings due to non-hydrogenic effects are small. In contrast $|M|=1$ states (which are also excited but with a far weaker efficiency at low fields) involve non-hydrogenic $P, D$ states. As seen in the figure, their Stark mixing begin with a quadratic (non-hydrogenic) perturbation of the levels position. The redistribution into the $|M|=1$ hydrogenic manifold results in strong interference profiles and large anticrossings. The field value is $E=2.37(\mathrm{~V} / \mathrm{cm})$. (The cell is not the one used for obtaining the data of Fig. 1 and 2). The squares, dots and small dots respectively indicate a strong line as in zero field conditions, a smaller line associated with the splitting into several components of the zero field one and a very small but reproducible line.

spectrum is concerned. This is quite clearly seen on the behaviour of the lines around $n=59,60$, 61. Their positions linearly depend on the $\mathbf{E}$ field strength on the whole range under investigation. Furthermore, no significant departures from this behaviour occur in the regime where adjacent manifolds are interacting. Individual lines belonging to the various manifolds can still be tracked without any ambiguity. The plot exhibits the Stark behaviour as will be seen on the hydrogen atom, under such conditions that the second-order term is nearly negligible. (The latte is indeed responsible for a small departure from the linear behaviour unambiguously appearing on the plot of Fig. 3). Indeed, such features are entirely confirmed by straightforward calculations taking the small quantum defects of the $F, G, \ldots$ states into account. No significant departure from the hydrogenic behaviour is ever seen, but for the fact that small anticrossing instead of crossings are the rule when adjacent manifolds being to merge. The calculated anticrossings have a maximum order of $20 \mathrm{MHz}$ and are not measurable under the present experimental conditions for $M=3$ states. They are the direct consequences of the breaking of the dynamical symmetry due to the nonCoulombic corrections to the Coulomb potential. Although the behaviour of the energies is, as expected, hydrogenic, the intensity features of the spectrum are more strongly depending on the non-Coulombic corrections to the potential.

In contrast, $|M|=1$ states strongly involve non-hydrogenic $\mathrm{P}, \mathrm{D}, \ldots$ states for which the quantum defects are non negligible at low fields. Consequently, as seen on figure 3, they first exhibit a (non-hydrogenic) second-order Stark effect at low fields. It is not exactly quadratic since it involves the coupling between numerous discrete states with $|M|=1$. Here the anticrossings are strong (in the range of hundredths $\mathrm{MHz}$ ) before recovering a quasi-linear behaviour at higher field values. This is confirmed through numerical simulations showing that the energy spectrum is irregular compared to that of $|M|=3$ states. 
As a matter of fact and compared to that of figure 3, a plot in $\pi$ polarization (in which non Coulombic states have a dominant role) does not exhibit any regular linear behaviour in this range of electric field, especially in the region of crossing of the manifolds.

\section{Conclusion.}

The experimental evidence of linear Stark patterns on an atomic Rydberg series studied under vapour phase conditions at extremely low electric field values has been reported. Previous experimental attempts have been performed with fields in the range $1-10 \mathrm{kV} / \mathrm{cm}$ [11], on low lying Rydberg states. As examplified here, the strong mixing regime can be reached at fairly low field values on high members of the series and a study of the building up of the Stark resonance spectra from the members of the Rydberg series seems to be within practical possibilities. Moreover, in this experiment using c.w. dye laser excitation, the lineshape of the laser is always wellcontrolled thus allowing a relevant further discussion of the intensity aspects of the spectrum. These aspects are far from being featureless and allow us to point out the role of a new class of Fano interference effects, between a discrete non-hydrogenic channel and a quasi-continuum of discrete states (those of the quasi-hydrogenic Stark manifold), in the transition between the nonhydrogenic and hydrogenic regimes of the Stark effect.

Finally, these studies of the linear Stark effect are a step towards a further study of the atomic spectrum in crossed $(\mathbf{E}, \boldsymbol{\beta})$ field conditions, when the joint actions of the paramagnetic interaction and the linear Stark effect give rise to a new signature of the spectrum [10,15]. The present experimental situation has been shown to be suitable for a study of the effects of each field separately $[12,13]$. It is likely that it will prove suitable too for studying their combined effects.

\section{References}

[1] Feneuille, S. and Jacquinot, P., Adv. Atom. Mol. Phys. 17 (1981) 99.

[2] Freeman, R. R., in Atomic Physics 7, D. Kleppner and F. M. Pipkin Ed. (Plenum Press) 1981.

[3] Косн, P. M., in Atomic Physics 7, D. Kleppner and F. M. Pipkin Ed. (Plenum Press) 1981.

[4] Feneullle, S., Liberman, S., Pinard, J. and Jacquinot, P., C. R. Hebd. Séan. Acad. Sci. 284 (1977) 291.

[5] Fréman, R. R. and Economou, N. P., Phys. Rev. A 20 (1979) 2356.

[6] Luk, T. S., Dimauro, L., Bergeman, T. and Metcalf, H., Phys. Rev. Lett. 47 (1981) 83.

[7] Luc-Koenig, E., Feneuille, S., Lecomte, J. M., Liberman, S., Pinard, J. and Taleb, A., Colloq. Intern. CNRS 334, J. Physique Colloq. 43 (1982) C2-153.

[8] Harmin, D., Phys. Rev. A 26 (1982) 2656.

[9] Feneuille, S., Liberman, S., Pinard, J. and Taleb, A., Phys. Rev. Lett. 42 (1979) 1404.

[10] Grozdanov, T. P. and Solovev, E. A., J. Phys. B 15 (1982) 1195.

[11] Littman, M. G., Zimmerman, M. L., Ducas, T. W., Freeman, R. R. and Kleppner, D., Phys. Rev. Lett. 36 (1976) 788.

Zimmerman, M. L., Littman, M. G., Kash, M. M. and Kleppner, D., Phys. Rev. A 20 (1979) 2251.

[12] Gay, J. C., Delande, D. and Biraben, F., J. Phys. B 13 (1980) L-729.

[13] Delande, D. and Gay, J. C., Phys. Lett. 82A (1981) 399.

[14] Collins, C. B., Johnson, B. W., Mirza, M. Y., Popescu, D. and Popescu, I., Phys. Rev. A 10 (1974) 813.

[15] Littman, M. G. and KorevaAr, E., Colloq. Intern. CNRS 334, J. Physique Colloq. 43 (1982) C2-455. 